\title{
USO DE BISFOSFONATOS E A OCORRÊNCIA DA OSTEONECROSE DOS MAXILARES: UMA REVISÃO DE LITERATURA
}

\author{
Victória Escóssia Germano ${ }^{\prime}$ \\ Alana Maria Gadelha de Medeiros I \\ Cindhi Mayra Rodrigues Xavier I \\ Denner Aires Mendonça Fonsecal \\ *Emanuelle Louyde Ferreira de Lima"
}

\section{RESUMO}

Os bisfosfonatos são uma classe de medicamentos utilizada nos serviços de saúde para o tratamento de doenças ósseas. Essas substâncias atuam nos osteoclastos, diminuindo a reabsorção e remodelamento ósseo. O uso dessa medicação pode levar a osteonecrose, uma patologia que se caracteriza pela necrose do tecido ósseo, devido a deficiência na irrigação sanguínea, podendo ocorrer em diversos ossos, inclusive nos maxilares. O presente trabalho, objetivou revisar a literatura existente sobre a relação entre o uso de bisfosfonatos e o risco de desenvolvimento de osteonecrose nos maxilares, tendo em vista que é um assunto muito importante para estabelecer a correta condução dos casos clínicos pelos profissionais da área médica e odontológica. Trata-se de uma revisão de literatura, com base em artigos, acerca do tema, encontrados no PubMed e que foram sintetizados e analisados. Foi visto que a possibilidade da ocorrência de osteonecrose, devido a prescrição ampla de bisfosfonatos e seu uso por longos períodos, vem tornando essencial o conhecimento das aplicações e suas consequências, pelas áreas responsáveis, a fim de evitar a ocorrência ou, pelo menos, minimizar os seus danos.

PALAVRAS-CHAVE: Osteonecrose. Bifosfonatos. Osteonecrose da Arcada Osseodentária Associada a Bifosfonatos.

Discente do curso do Bacharelado em Odontologia da Faculdade Nova Esperança de Mossoró, FACENE/ I RN. Departamento de Odontologia. CEP 59617-260, Mossoró, Rio Grande do Norte, Brasil. ORCID ID: 0000-0001-7479-5297; 0000-0003-1045-2919; 0000-0003-4452-2698; 0000-0002-9441-7100

Mestra, Docente da Faculdade Nova Esperança de Mossoró, FACENE/RN. Departamento de Odontologia. CEP 59617-260, Mossoró, Rio Grande do Norte, Brasil.

*Autor correspondente: emanuellelouyde@facenemossoro.com.br Orcid: 0000-0003-2341-7565 


\section{INTRODUÇÃO}

Os bisfosfonatos são uma classe de medicamentos análoga ao pirosfosfato utilizada, tanto na atenção básica de saúde, quanto em atendimentos especializados, para o tratamento de doenças que afetam o metabolismo ósseo. Seu uso visa a estabilização da perda óssea, consequentemente, diminuindo o risco de fraturas vertebrais, ou de ossos longos. Porém, sua excessiva reabsorção é associada à osteoporose, a doença de Paget, ou a conduta clínica para a hipercalcemia severa relacionada a fatores de malignidade, metástase e mieloma múltiplo. Essas substâncias atuam nos osteoclastos, diminuindo a reabsorção e remodelamento ósseo, além de agir inibindo os mediadores da inflamação, essenciais na recuperação e manutenção da matriz óssea. ${ }^{1,2}$

Embora apresentem resultados excelentes no tratamento de doenças ósseas, o uso dos bisfosfonatos, a longo prazo, normalmente com mais prevalência quando administrado por via intravenosa, com aplicação mensal e por um período maior que três anos, pode predispor à osteonecrose da mandíbula e maxila, sendo mais frequente na mandíbula e em locais que sofreram extrações de elementos dentários. ${ }^{1}$

A osteonecrose pode ser considerada como a incapacidade do tecido ósseo, afetado, em se reparar e se remodelar, quando exposto a fatores desencadeantes, normalmente associados a exodontias, estresse mecânico, infecção dental e periodontal. ${ }^{4}$ Muitas vezes, o seu desenvolvimento está relacionado ao uso de bisfosfonatos, mesmo que sem causa específica direta. Algumas hipóteses cogitam a influência desses fármacos sobre a distribuição do suprimento sanguíneo ósseo, bem como a possibilidade da ocorrência ser provada pelo desenvolvimento de osteomielite crônica. $^{3}$

Os processos exatos quanto a sua patogênese ainda não são claros, existindo, atualmente, duas teorias para explicar o seu mecanismo. A inibição da remodelação óssea e inibição da angiogênese capilar. ${ }^{4}$

No primeiro caso, a rotatividade das células ósseas (produção, distribuição e apoptose) é diminuída com o uso prolongado dos bisfosfonatos, provocando danos persistentes na integridade óssea, acumulandoos e, por consequência, resultando em uma estrutura com baixa competência

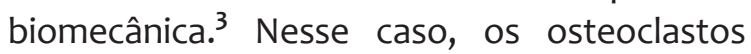
têm sua atividade reduzida, não reabsorvendo a matriz mineral oriunda da apoptose dos osteoblastos e osteócitos, que vivem em média 150 dias. $^{5}$

A ineficiência ou, em alguns casos, até mesmo inexistência deste fenômeno inviabiliza a liberação de citocinas da proteína morfogenética óssea (BMP) e do fator de crescimento, semelhante a insulina (IGF), responsável pela indução da diferenciação das células mesenquimais, tornando os túbulos responsáveis pela passagem de vasos sanguíneos e células nervosas, também chamado de Osteon ou Sistema de Harvers, acelular e necrótico2. Por fim, os pequenos vasos capilares encontrados no osso evoluem, tornando-o avascular e, portanto, mais suscetível à fraturas. ${ }^{2,6}$

A segunda teoria da patogênese sugere que, apartirdautilização das drogas daclassedos bisfosfonatos, principalmente o pamidronato e o ácido zoledrônico, inibe-se a angiogênese capilar, responsável pelo desenvolvimento de novos vasos sanguíneos, acarretando diminuição na formação de túbulos capilares e retração do fator de crescimento endotelial. Portanto, de acordo com essa teoria, há uma diminuição da quantidade de vasos sanguíneos devido a inibição da angiogênese capilar, 
resultando em uma necrose avascular do osso. ${ }^{6}$

Diante disso, o presente estudo objetivou revisar na literatura existente artigos que tratem acerca da relação entre o uso de bifosfonatos e o risco de

\section{MATERIAL E MÉTODOS}

O presente trabalho é uma revisão de literatura, com base em artigos encontrados no PubMed, sintetizados e analisados, através dos conceitos-chave da pesquisa, como "osteonecrose" e "bisfosfonatos". Foram selecionados 20 artigos nas línguas portuguesa, inglesa e espanhola, publicados entre os anos de 2015 e 2020, sendo priorizados os estudos epidemiológicos e revisões de literatura.

Foram incluídos no trabalho textos de pesquisa ou revisão que abordassem o tema e fornecessem informações acerca dos

\section{RESULTADOS E DISCUSSÃO}

A prescrição dos bisfosfonatos é comum a ponto de inúmeros estudos surgirem em variadas partes do mundo e com indivíduos em circunstâncias tanto generalistas quanto específicas. Sendo assim, estudos extensos apresentam resultados bastante confirmatórios com o que já se especula há alguns anos, desde a descoberta da relação entre os bisfosfonatos e a osteonecrose de maxilares. $O$ estudo em questão utilizou mais de 500 mil pacientes, especificamente mulheres após a menopausa (entre a sexta e a sétima década de vida), que utilizavam de medicação oral com bisfosfonatos. Foi percebido que o risco deste grupo apresentar osteonecrose de maxilares aumentava em 6 vezes, comparando-se às pessoas que nunca haviam tomado. Também se deu a percepção de que a medicação intravenosa está desenvolvimento de osteonecrose nos maxilares dos pacientes, tendo em vista a importância desse conhecimento para a correta condução dos casos clínicos pelos profissionais da área médica e odontológica.

mecanismos de ação dos bisfosfonatos e seus efeitos, quando relacionados a osteonecrose dos maxilares dentro do limite dos anos de publicação. Já como critério de exclusão, artigos com mais de 5 anos de publicação, ou com enfoque voltado para a área de implante e seu sucesso, quando relacionado ao uso do medicamento, foram excluídos. Sendo assim, dos selecionados, foi separada inicialmente uma coletânea de artigos para serem trabalhados individualmente pelos autores.

extremamente associada a osteonecrose, com cerca de 7-12\% de acometimento para usuários com um risco crescente, conforme o seu tempo de utilização. Em contrapartida, a utilização de medicação de forma oral, embora relacionada com a osteonecrose se apresenta muito mais branda, quando comparada a endovenosa, podendo ser até 5 vezes menor e necessitar de muito mais tempo de administração. ${ }^{7}$

Outro estudo obteve resultados próximos mesmo com um espaço amostral menor, utilizando 20 pacientes sendo 13 mulheres e 7 homens, todos na sétima década de vida e com histórico de administração de Zolentronate endovenosa em 19 deles. Além dos dados semelhantes, foi notável também a associação da osteonecrose com a imunidade mais baixa, principalmente quando o bisfosfonato está associado a uma medicação 
do tipo corticosteróide, com acometimento maior em maxila. A medicação oral também foi menos relacionada a osteonecrose segundo esse mesmo estudo. ${ }^{8}$ Já um levantamento realizado em ratos de laboratório, apresentou que o uso oncológico dos bisfosfonatos, muito comumente endovenoso, possui uma prevalência ainda maior de osteonecrose, principalmente quando há presença de inflamações orais (mesmo as que não são relacionadas com cirurgia bucal) que, segundo a pesquisa, seriam facilitadores do processo de necrose, algo visualizado em ratos que também tinham periodontite. ${ }^{9}$

No entanto, há estudo que demonstra uma incidência um pouco mais baixa utilizando um outro medicamento, o Bevacizumab. Neste, a ocorrência permaneceu entre $0,3 \%$ a 0,4\% em uma análise com 3560 pacientes, demonstrando que, não necessariamente, todos os medicamentos que utilizam bisfosfonatos irão apresentar grandes reações. Além disso, este mesmo estudo demonstrou que há, aparentemente, irregularidades quanto a consistência na relação tempo de utilização do medicamento e dose. Enquanto outros relatos denunciam uma atuação mais consistente com utilizações mais longas, este apresenta uma variação considerável entre os pacientes, uma vez que eles apresentavam a necrose de 2 a até 15 meses de utilização da medicação. Mas, o próprio artigo relata que podem haver fatores simultâneos que prejudiquem essa interpretação. ${ }^{10}$

Há também estudos que relatam incidências ainda mais baixas. Em uma população de 13 mil pacientes havia apenas $0,1 \%$ com um crescimento para 0,21\% em tratamentos com mais de 4 anos. $\mathrm{Na}$ Escócia, um estudo com 90 mil pacientes obteve uma incidência com apenas 0,004\%. $\mathrm{E}$, dentro dessa margem, foi visualizado que apenas os indivíduos com ulcerações orais provenientes de outras medicações desenvolveram as ulcerações. ${ }^{11}$

Portanto, embora grande parte dos estudos apontem para uma incidência mais incisiva do que outras, aparentemente há circunstâncias específicas que corroboram para uma maior osteonecrose nos maxilares como as inflamações e ulcerações orais, apontadas como decorrentes, em maior parte, de outras medicações utilizadas para o tratamento desse paciente, como corticoesteróides ${ }^{11}$

\section{A PATOGÊNESE DA OSTEONECROSE}

\section{Relação entre o bisfosfonatos e osteonecrose dos maxilares}

O uso de bisfosfonatos tem sido relacionado com $\mathrm{O}$ desenvolvimento de osteonecrose da mandíbula. O termo BRONJ (Osteonecrose da Mandíbula Associada ao Bisfosfonato) se refere a exposição de osso na região maxilofacial que persiste por mais de 8 semanas em indivíduos que fazem uso de bisfosfonatos e não foram submetidos a radiação. Junto com a exposição óssea podem ser observados outros sintomas e sinais como, dor, inchaço, infecção, fístulas orocutâneas, abscessos, mobilidade dental, perda de elementos dentários, halitose, dificuldade na mastigação e desfiguração facial. Em 4,5\% dos casos, dependendo da região e do estágio da necrose, também pode ocorrer fratura da mandíbula. ${ }^{12}$

A causa da osteonecrose, associada ao uso de bisfosfonatos, permanece uma incógnita. Uma hipótese seria que 
os bisfosfonatos afetam a distribuição do suprimento sanguíneo do osso por inibição do crescimento do endotélio vascular. ${ }^{4}$ Novas teorias sugerem que a osteonecrose é provocada em decorrência do desenvolvimento de osteomielite

\section{Fatores de risco e contraindicações}

A incidência de BRONJ é relatada mais frequentemente em pacientes que fazem uso de altas doses dessa medicação por via intravenosa e durante longos períodos, em geral mais de 4 anos. Esse período pode variar de acordo com o medicamento utilizado. Por exemplo, a administração intravenosa de zoledronato, por um período superior a um ano e meio, pode levar ao desenvolvimento de BRONJ, enquanto em uma terapia à base de alendronato via oral esse período se estenderia para quatro anos e seis meses, em média. ${ }^{14}$

A BRONJ é mais comum em indivíduos que fazem o uso de bisfosfonatos para o tratamento de neoplasias malignas e controle de metástases ósseas. Já incidência de osteonecrose nos pacientes em tratamento paraosteoporoseébemmenor, principalmente devido a via de administração. ${ }^{15}$

Entre os fatores de risco para o desenvolvimento de BRONJ estão procedimentos odontológicos invasivos como, por exemplo, a extração dentária prévia, justamente porque, em casos de extração,

\section{Tratamento e recomendações}

Antes do início da terapia com bisfosfonatos é indicado que o indivíduo passe por uma avaliação dental para realizar qualquer procedimento necessário e eliminar fontes de infecção. Após exodontias, ou crônica. Bactérias dos gêneros Actinomyces e Staphylococcus contaminam e lesam o osso que, devido ao uso de bisfosfonatos, e, consequente diminuição da capacidade de reabsorção óssea, não consegue remover o tecido infectado com a rapidez necessária. ${ }^{2}$

ocorre a exposição óssea. Geralmente o fechamento do espaço se dá por segunda intenção, ou seja, o processo de reparo é mais complicado e demorado, tornando o local mais propício a infecções que possam contribuir para a ocorrência da osteonecrose. ${ }^{16}$

Além disso, outros fatores podem estar associados como doenças periodontais, uso concomitante de corticosteróides, quimioterapia, terapia imunossupressora, tabagismo, idade e comorbidades médicas como, obesidade, diabetes mellitus, hipertensão, hipotireoidismo, insuficiência renal. ${ }^{17}$

Entretanto, observações indicam que extrações dentárias são um fator determinante para a ocorrência de BRONJ em $67 \%$ dos casos. Visto que uma revisão de 114 casos analisados na Austrália mostrou que $73 \%$ dos casos ocorreram após as extrações. A frequência em pacientes com osteoporose foi de $0,01 \%$ - 0,04\% e se ocorreu a extração dental foi de 0,9\% - 0,34\%. Em pacientes com metástases ósseas a incidência foi de 0,33\% $1,15 \%$ e após extrações foi de $6,7 \%-9,1 \%{ }^{18}$ 
Após o diagnóstico de BRONJ, recomenda-se a suspensão do uso de bisfosfonato. A avaliação do caso de cada paciente, sua expectativa de vida e presença de comorbidades são determinantes para definir o tratamento adequado. Osteoctomias segmentais para a remoção do tecido necrótico são indicados apenas para

\section{CONSIDERAÇÕES FINAIS}

A partir desta pesquisa, constata-se que, emboragrandeparte dos estudos apontem para uma incidência muito maior do que outros, há circunstâncias específicas desconhecidas, pois, aparentemente, há tratamentos muito mais esclarecidos e constantes, na maior parte dos estudos, do que os fatores de risco e as influências na incidência.

Torna-se visível que essas divergências nas estatísticas são reflexos de componentes variáveis ainda não muito claros, como a relação do paciente tratado com bisfosfonatos, associados a outras medicações como corticosteróides que podem, por exemplo, casos severos e se o indivíduo apresentar expectativa de vida razoável. ${ }^{17}$ Caso contrário, recomenda-se tratamento sintomático e conservativos, que inclui o uso de antibióticos como clindamicina (1200 mg por dia, durante 14 dias), abandonar o uso de próteses, adoção de uma alimentação leve ou, em casos mais críticos, tubo nasogástrico. ${ }^{18}$ causar algum tipo de dano a mucosa oral de forma consistente, ou reduzir a capacidade imunológica do paciente, sendo, talvez, um fator facilitador para a osteonecrose.

Sendo assim, é extremamente necessária a investigação acerca dessa discrepância entre dados e quais são as situações dos pacientes que resultaram nessa diferenciação no acometimento para, dessa forma, traçar conjuntos medicamentosos diferenciados para os indivíduos tratados com bisfosfonatos, ou até redirecionar a atenção para outras abordagens clínicas.

\title{
BISPHOSPHONATE USE AND OCCURRENCE OF JAW OSTEONECROSIS - A LITERATURE REVIEW
}

\begin{abstract}
Bisphosphonates are a class of medicines used in health services to treat bone diseases. These substances act on osteoclasts, reducing bone resorption and remodeling. The use of this medication can lead to osteonecrosis, a condition that is shown by bone tissue necrosis due to damage to blood supply and can occur in many bones, including the jaw. To review existing literature on the relationship between bisphosphonate use and risk of developing jaw osteonecrosis bearing in mind that it is an extremely important issue to define the correct correction of clinical cases by professionals in the field. This study deals with a literature review, based on articles found in PubMed, synthesized and analyzed on the subject. The possibility of occurrence of osteonecrosis due to the wide prescription of bisphosphonates and its use for long periods, it is essential to know the applications and their consequences for the areas used, in order to avoid the occurrence or at least the damage caused.
\end{abstract}

KEYWORDS: Osteonecrosis. Diphosphonates. Bisphosphonate-Associated Osteonecrosis of the Jaw. 


\section{REFERÊNCIAS}

1. Jesus AP, Filho FSS, Cardoso JA, Câncio AV, Simões CC, Farias J. Tratamento cirúrgico para osteonecrose dos maxilares induzida por bisfosfonatos: relatos de casos. RFO-UPF, 2019; 24(1): 22-30.

2. Lima BKS, Branco SJSC, Fontes VC, Pinheiro AJMCR, Neto VHLGM, Zago PMW. Perfil microbiológico bucal dos pacientes portadores de osteonecrose maxilar induzida por bisfosfonatos. Rev Investigação Biomédica, 2017; 9(2): 187-97.

3. Coléte JZ, Hadad H, Momesso GAC, Filho HS, Fernandes BR, Júnior IRG. Implantes em pacientes com osteonecrose dos maxilares associado ao uso de bifosfonatos. Relato de caso e revisão de literatura. Arch Health Invest, 2019; 8(1): 20-27.

4. Souza TB. Osteonecrose dos maxilares induzida por bisfosfonatos intravenosos. Revista Cathedral, 2020; 2(1): 1-11.

5. Júnior AACP. Osteonecrose dos maxilares associada ao uso de bisfosfonatos. CEP, 2017; 31(1): 404-07.

6. Chaves RAC, Queiroz TP; Faloni APS. Bifosfonatos e Denosumabes: mecanismos de ação e algumas implicações para a implantodontia. RebraM, 2018; 21(2): 66-80.

7. Wotton $\mathrm{CJ}$ et al. Use of oral bisphosphonates and risk of hospital admission with osteonecrosis of the jaw: large prospective cohort study in UK women. Bone, 2019; 124: 69-74.

8. Lungu $A E$, Lazar MA, Tonea $A$, Rotaru $H$, Roman RC, Badea ME. Observational study of the bisphosphonate-related osteonecrosis of jaws. Clujul Medical, 2018; 91(2): 209.
9. Messer JG et al. Zoledronate treatment duration is linked to bisphosphonate-related osteonecrosis of the jaw prevalence in rice rats with generalized periodontitis. Oral diseases, 2019; 25(4): 1116-35.

10. Guarneri V, Miles D, Robert N, Diéras V, Glaspy J, Smith I, et al. Bevacizumab and osteonecrosis of the jaw: incidence and association with bisphosphonate therapy in three large prospective trials in advanced breast cancer. Breast cancer research and treatment, 2010; 122(1): 181-88.

11. Ilyas Z, Camacho PM. Rare adverse effects of bisphosphonate therapy. Current Opinion in Endocrinology, Diabetes and Obesity, 2019; 26(6): 335-38.

12. Rollason V, Laverrière A, MacDonald LC, Walsh $T$, Tramèr MR, Vogt-Ferrier NB. Interventions for treating bisphosphonate-related osteonecrosis of the jaw (BRONJ). Cochrane Database of Systematic Reviews, 2016 (2): 1-30.

13. Caminha RD, Chicrala GM, Júnior LAVS, Santos PSS. Perfil de risco para osteonecrose dos maxilares associada a agentes antiangiogênicos. Einstein, 2019; 17(3): 1-7.

14. De Sousa AS, Almeida VP, Taisa J, Savedra LF, Rodrigues IV, Giro G.. Protocolo de atendimento odontológico de pacientes em tratamento com bisfosfonatos. Rev Saúde-UNG-Ser, 2019; 12(1/2): 54-61.

15. Peer A, Khamaise M. Diabetes as a risk factor for medication-related osteonecrosis of the jaw. Journal of dental research, 2015; 94 (2): 252-60.

16. Khan AA et al. Diagnosis and management of osteonecrosis of the jaw: a systematic review and international consensus. Journal of Bone a 


\section{revista de
ciências \\ da saúde ESPERANÇA}

nd Mineral Research, 2015; 30(1):3-23.

17. Bodem JP, Kargus S, Engel M, Hoffmann J, Freudlsperger C..Valueofnonsurgicaltherapeutic management of stage I bisphosphonate-related osteonecrosis of the jaw. Journal Of Craniomaxillofacial Surgery, 2015; 43(7): 1139-43.
VOLUME 18 - NÚMERO 2 - Ago/2020 ISSN ELETRÔNICO 2317-7160

134

18. Rodrígues-Sanchéz MDP. The Effectiveness of the Low-Level Laser, Antibiotic and Surgical Therapy in the Treatment of Medication-Related Osteonecrosis of the Jaws: A Case Report. Journal of Lasers in Medical Sciences, 2020; 11(1): 98-03. 